\title{
Inclusive Inelastic Electron Scattering from Nuclei
}

\author{
Nadia Fomin \\ University of Virginia, Charlottesville, VA
}

\begin{abstract}
Inclusive electron scattering from nuclei at large $\mathrm{x}$ and $Q^{2}$ is the result of a reaction mechanism that includes both quasi-elastic scattering from nucleons and deep inelastic scattering from the quark consitituents of the nucleons. Data in this regime can be used to study a wide variety of topics, including the extraction of nuclear momentum distributions, the influence of final state interactions and the approach to $y$-scaling, the strength of nucleon-nucleon correlations, and the approach to $x$ - scaling, to name a few. Selected results from the recent experiment E02-019 at the Thomas Jefferson National Accelerator Facility will be shown and their relevance discussed.
\end{abstract}

Keywords: scaling, electron scattering, momentum distributions, SRCs

PACS: $21.30-\mathrm{x}$

\section{SCALING IN THE QUASIELASTIC REGIME}

The quasielastic cross section is analyzed in the Plane Wave Impulse Approximation (PWIA), where the dominant process is assumed to be scattering from an individual nucleon, whose motion is treated relativistically. The inclusive cross section for this process is given by [1]

$$
\frac{d^{2} \sigma}{d \Omega d E}=\sum_{N=1}^{A} \int d E \int d^{3} p S(p, E) \sigma_{e N} \delta\left(E_{0}+v+\sqrt{M_{A-1}^{* 2}+(\vec{p}+\vec{q})^{2}}\right)
$$

where $S(p, E)$ is the nucleon spectral function, $\sigma_{e N}$ is the off-shell electron-nucleon cross section. Following the steps of [1], we can simplify and factorize this expression to give us:

$$
\frac{d^{2} \sigma}{d \Omega d E}=F(y, q) \frac{1}{Z \sigma_{e p}+N \sigma_{e n}} \frac{\mathbf{q}}{\sqrt{M^{2}+(\mathbf{p}+\mathbf{q})^{2}}} .
$$

$\mathrm{F}(\mathrm{y}, \mathrm{q})$ is the scaling function defined as

$$
F(y, q)=2 \pi \int_{E_{s}^{m i n}}^{\infty} d E \int_{y 1\left(E_{s}^{m i n}\right)}^{\infty} d p p S(p, E)
$$

where $y$ is the longitudinal momentum of the struck nucleon, determined by the argument of the $\delta$-function in Eq. 1. Expression 2 allows us to extract the response function $\mathrm{F}(\mathrm{y}, \mathrm{q})$ from the measured cross section. Scaling of the reduced cross sections $(\mathrm{F}(\mathrm{y}, \mathrm{q}))$ in $y$ has been observed [8, 6] in the quasi-elastic region (Example: Fig. 1). This means that the reduced cross section, instead of being a function of energy and momentum transfers, instead depends on only one variable, in this case $y$, and the reduced cross sections for different kinematics all lie on one scaling curve. 

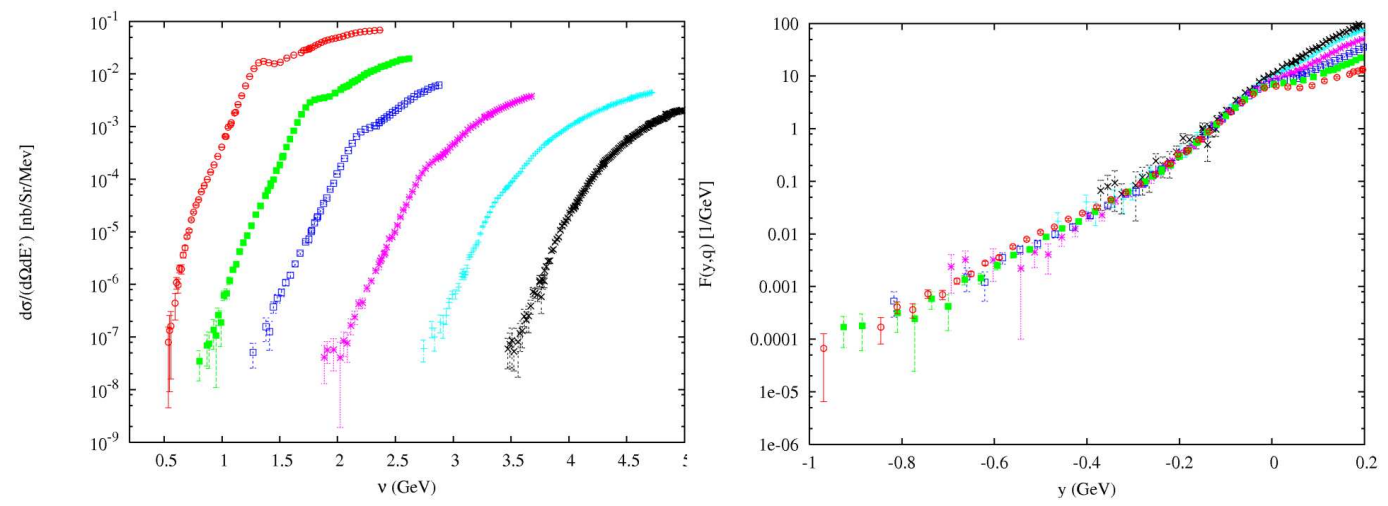

FIGURE 1. Left: ${ }^{3} \mathrm{He}$ Cross sections for 6 scattering angles $\left(18^{\circ}-50^{\circ}\right)$ as a function of $v$, energy loss, at $5.766 \mathrm{GeV}$ (JLab experiment E02-019). Right: The scaling functions extracted from the above cross sections as a function of $y$, the longitudinal momentum of the struck nucleon. $Q^{2}$ range covered is 2.5-7.4 at the quasielastic peak. Scaling is observed for negative values of $y$, corresponding to the $x>1$ region, where quasielastic contributions are dominant.

The scaling function can be related to the nucleon momentum distribution, but there are a few obstacles. First, there's a binding correction that comes from the possibility of having the recoil nucleus be in an excited state. Also, the PWIA approach does not take Final State Interactions (FSIs) into account, whose contributions are largest at low values of $Q^{2}$ and large negative $y$ 's and enough to change the predicted approach to scaling. And finally, in order to extract a momentum distribution, one needs to be able to subtract or reasonably neglect any contributions from inelastic processes which requires either a cross-section model or data taken at kinematics where inelastic processes don't contribute.

\section{SHORT RANGE CORRELATIONS}

In Fig. 1, we observe the struck nucleons of very high momenta. The repulsive NN force imparts high momentum to the nucleons as the interaction distance between the nucleons becomes smaller than the average inter-nucleon spacing. The momentum of fast nucleons is balanced by the correlated nucleon(s), rather than the rest of the nucleons.

The ideal regime for studying SRCs is at $x>1$ and $Q^{2}>1$, where scattering from lowmomentum nucleons is suppressed and the energy transfer is higher than the kinetic energies of the correlated nucleons. Here, the mean field contribution is negligible, and the inclusive cross section can be approximated with [4]

$$
\sigma_{A}\left(x, Q^{2}\right)=\sum_{j=2}^{A} \frac{a_{k}(A)}{j} \sigma_{j}\left(x, Q^{2}\right)
$$

where $\sigma_{A}\left(x, Q^{2}\right)$ is the electron-nucleus cross section, $\sigma_{j}\left(x, Q^{2}\right)$ is the electron- $j$ nucleon-correlation cross section, and $a_{j}(A)$ is proportional to the probability of finding 

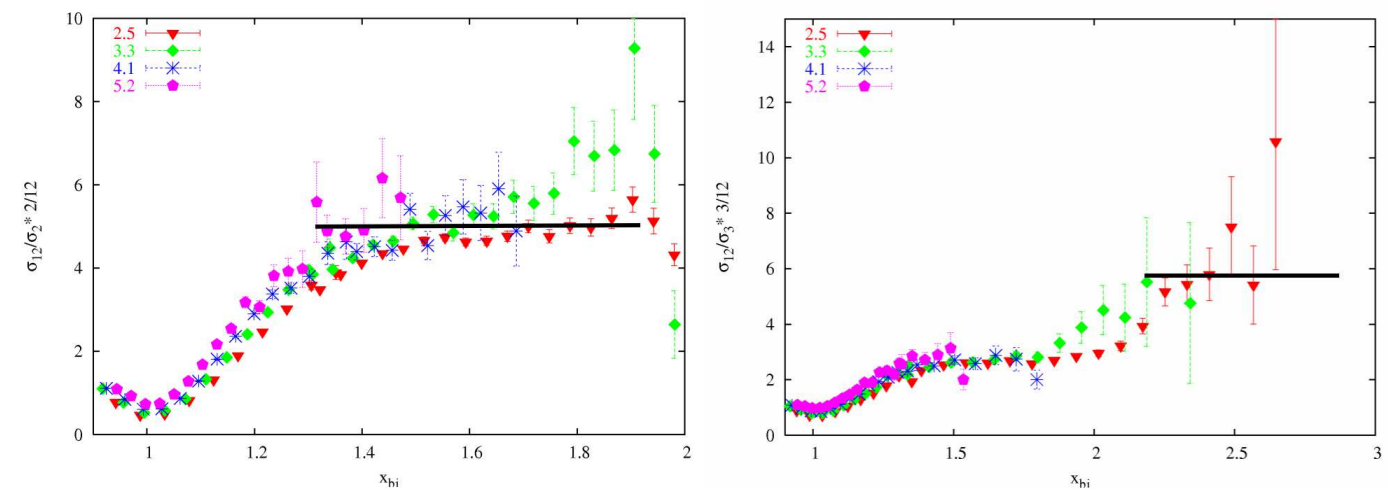

FIGURE 2. SRC ratios for ${ }^{12} \mathrm{C}$. The left plot shows the ratio to ${ }^{2} \mathrm{H}$ and the right plot shows the ratio to ${ }^{3} \mathrm{He}$. The ratio for $1.4<x<2$ is proportional to the abundance of the NN correlations in Carbon, and the ratio for $\mathrm{x}>2.4$ is proportional to the abundance of $3 \mathrm{~N}$ correlations.

a nucleon in a $j$-nucleon correlation. Using ${ }^{2} \mathrm{H}$ and ${ }^{3} \mathrm{He}$ cross sections as well as a theoretical calculation for the fraction of nucleons in each of those that are in a 2- or 3-N correlation, one can obtain SRCs for heavier targets, which show the abundance of correlations. For example, for ${ }^{12} \mathrm{C}$, shown in Fig. 2 has a $\approx 20 \%$ and $\approx 0.6 \%$ probability of a $2 \mathrm{~N}$ and $3 \mathrm{~N}$ SRC, respectively, which is in agreement with previous measurements [7].

\section{INELASTIC SCATTERING}

As one can see from Fig. 1, $y$-scaling fails for $y>0$, where inelastic processes dominate. In this region, the cross section is described in terms of the nuclear structure functions:

$$
\frac{d^{2} \sigma}{d \Omega d E}=\sigma_{m o t t}\left[W_{2}^{A}(\mathbf{q}, v)+2 W_{1}^{A}(\mathbf{q}, v) \tan ^{2}(\theta / 2)\right]
$$

In the limit of high energy loss and momentum transfer in the DIS regime, the structure functions simplify to functions of $x$ alone. When one examines the structure functions with the Nachtmann variable $\xi$ [2], which extends the scaling to lower values of $Q^{2}$, one expects to see the same scaling behavior as is seen for $x$ in the same kinematic region. $\xi$ reduces to $x$ as $Q^{2} \rightarrow \infty\left(\xi=2 x /\left(1+\sqrt{1+4 M^{2} x^{2} / Q^{2}}\right)\right.$.

Our analysis of the inelastic cross section is done through the study of the $v W_{2}^{A}$ structure function, which can be extracted from the measured cross section in the following way:

$$
v W_{2}^{A}=\frac{d^{2} \sigma}{d \Omega d E} \cdot \frac{v}{\sigma_{m o t t}\left[1+2 \tan ^{2}(\theta / s) \frac{1+v^{2} / Q^{2}}{1+R}\right]},
$$

where $R$ is the ratio of the longitudinal cross section to the transverse [9].

Fig. 3 shows that the $v W_{2}^{A}$ structure function scales in $x$ in the DIS region. Since the $\xi$ variable is analogous to $x$, we expect $v W_{2}$ to scale in $\xi$ as well, in this region. However, scaling is observed for all values of $\xi$, including the quasielastic region. 

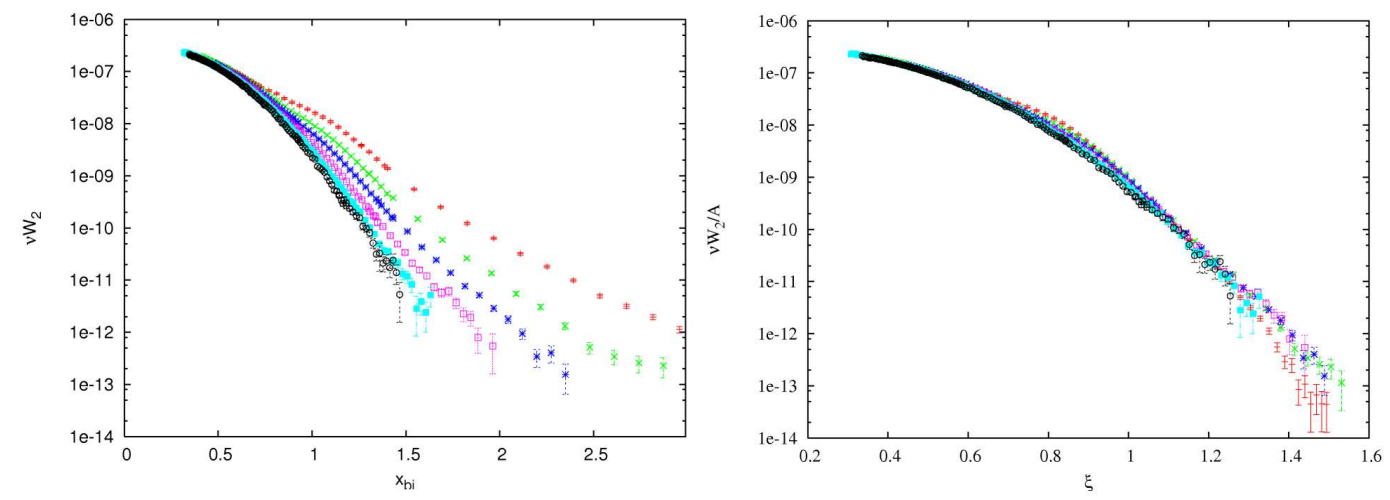

FIGURE 3. Structure function $v W_{2}^{A}$ for ${ }^{12} C$ as a function of $x$ (left) and $\xi$ (right). Scaling is only observed for low values of $x$, but for all values of $\xi$, rather than just in the inelastic region.

The first explanation [3] of this observation involves expanding $\xi$ as a function of $y$, suggesting that the scaling we see in the quasielastic region is the same as the $y$ scaling analysis yields, with the presence of FSIs masked by the relationship between the two variables. Another explanation [10] states that the observed scaling is purely accidental and is just a result of the inelastic contribution falling off at the same rate as the quasielastic contribution rises.

A final explanation for this observation is that it might be due to local duality [5]. Bloom and Gilman observed that the structure function has the same $Q^{2}$ behavior as the resonance form factors. In fact, the scaling curve is recovered by averaging over the resonance peaks.

\section{CONCLUSION}

Despite the fact that the contributions from different processes measured in inclusive electron scattering are difficult to separate, it still offers a wealth of information. With a good sample of data over a variety of targets and kinematics, we can study nucleon momentum distributions, SRCs, scaling, $Q^{2}$ behavior of the $v W_{2}^{A}$ structure functions, as well as other physics.

\section{REFERENCES}

1. C. Ciofi degli Atti, E. Pace, and G. Salme, Phys. Rev. C43 (1991) 1155.

2. O. Nachtmann, Nucl. Phys. B63 (1973) 237.

3. O. Benhar and S. Liuti, Phys. Lett. B358 (1995) 173.

4. L. L. Frankfurt, M.I. Strikman, D.B. Day, and M. Sargsian, Phys. Rev. C48 (1993) 2451.

5. E.D. Bloom and F.J. Gilman, Phys. Rev. D4 (1971) 2901.

6. J. Arrington et al., Phys. Rev. Lett. 82 (1999) 2056.

7. K.S. Egiyan et al., Phys. Rev. Lett. 96 (2006) 082501.

8. D.B. Day et al., Phys. Rev. C48 (1993) 1849.

9. P.E. Bosted et al., Phys. Rev. C46 (1992) 2505.

10. D.B. Day and I. Sick, Phys. Rev. C69 (2004) 028501. 